\title{
Variations
}

Variations

Revue internationale de théorie critique

$23 \mid 2020$

Pour une gauche érotique

\section{Retour à Reims : une pièce à conviction?}

Fabien Granjon

\section{(2) OpenEdition}

Journals

Édition électronique

URL : http://journals.openedition.org/variations/1832

DOI : 10.4000/variations.1832

ISSN : 1968-3960

Éditeur

Les amis de Variations

Référence électronique

Fabien Granjon, «Retour à Reims : une pièce à conviction? 》, Variations [En ligne], 23 | 2020, mis en ligne le 01 septembre 2020, consulté le 07 septembre 2020. URL : http://journals.openedition.org/ variations/1832; DOI : https://doi.org/10.4000/variations.1832

Ce document a été généré automatiquement le 7 septembre 2020

Les ami•e•s de Variations 


\title{
Retour à Reims : une pièce à conviction?
}

\author{
Fabien Granjon
}

\section{NOTE DE L'ÉDITEUR}

À l'occasion, en janvier dernier, de l'adaptation à la scène par Thomas Ostermeier du best seller sociologique de Didier Eribon, Retour à Reims (2009), Fabien Granjon revient sur ce qu'il estime être à l'origine de ce « raté théâtral » : l'ambivalence des propos d'origine. L'auto-socioanalyse de l'universitaire amiénois propose en effet un intéressant exercice de retour sur soi, mais pêche par des analyses discutables du monde ouvrier et que le metteur en scène allemand peine à dépasser.

« La famille, c'est comme la merde : plus c'est proche, plus ça pue. » Giacinto Mazatella in Brutti, sporchi e cattivi (Ettore Scola) «Mon succès se mesurait à la distance que j'avais placée entre nous » John Edgar Wideman, Suis-je le gardien de mon frère?

1 Depuis 1999, Thomas Ostermeier dirige la Schaubühne am Lehniner Platz, (jusqu'en 2005 avec Sasha Waltz, danseuse et chorégraphe allemande), prestigieuse institution berlinoise dédiée au spectacle vivant. Metteur en scène très en vue, multipliant les collaborations à l'échelle internationale (Russie, États-Unis, Canada, Angleterre, etc.) et particulièrement en France (artiste associé au festival d'Avignon, au TNB, etc., il est également président du Haut conseil culturel franco-allemand), ses spectacles tirés du répertoire classique (Brecht, Büchner, Ibsen, Shakespeare, Tchekhov, etc.) tout comme du théâtre le plus contemporain (Kane, Dresser, Reza, etc.) sont, la plupart du temps, salués, voire encensés par la critique.

2 En 2016, le metteur en scène allemand découvre le livre de Didier Eribon, Retour à Reims (2009), qui vient d'être traduit et publié aux éditions Suhrkamp sous le titre Rückkehr 
nach Reims ${ }^{1}$. Il adapte l'ouvrage rapidement avec la complicité de Nina Wetzel (costumière et scénographe) et en fait une pièce qui se joue d'abord en anglais, avec Nina Hoss dans le rôle principal, l'une de ses comédiennes fétiches (la première a lieu en juillet 2017 au Manchester International Festival). Quelques mois plus tard, la pièce est jouée en allemand à la Schaubühne et enfin en français: d'abord en Suisse, au Théâtre Vidy-Lausanne, puis à Paris début 2019, à l'Espace Cardin-Théâtre de la ville². Ostermeier affirme avoir été particulièrement touché par le travail de l'essayiste français qui revient, dans son livre, sur ses origines sociales et la honte qu'il a longtemps éprouvé à l'égard du milieu ouvrier qui fut le sien (sentiment qui perdure), avant de partir à Paris pour y poursuivre des études supérieures et fréquenter d'autres espaces sociaux, dont les milieux intellectuels et gay. Lui-même issu des classes populaires (fils d'un soldat et d'une vendeuse) ayant traversé certaines des mêmes affres (« l'alcoolisme et la violence à la maison, j'ai aussi connu ça »; « C'était un milieu où chaque sou comptait. Comme Didier Eribon, j'ai vécu cette rupture avec ma famille. Et, comme lui, une fois arrivé dans une grande ville, j'ai dissimulé mes origines. J'avais honte de venir d'un milieu social inférieur $\left.{ }^{3} »\right)$ et ayant emprunté, sous certains aspects, une trajectoire biographique sensiblement analogue à celle d'Eribon, l'adaptation que Ostermeier propose du best-seller lui donne l'occasion de se dire par procuration, mais aussi celle de traiter une thématique qui lui semble politiquement des plus importantes: " la montée de l'extrême droite et l'échec de la gauche »: "ce livre constitue un matériau idéal pour parler de ce problème ${ }^{4} »$. Les représentations parisiennes vont donner lieu à des critiques pour le moins contrastées. Quand d'aucuns estiment, qu'avec cette pièce, Ostermeier "réinvente le théâtre politique ${ }^{5}$ " (rien de moins!), d'autres n'y ont vu qu' « une vulgaire pièce de boulevard », n'y ont entendu qu'une lecture " amorphe et bourgeoise » et ont vilipendé « une écriture dramatique qui rappellerait presque la qualité des téléfilms d'Antenne $2 »$.

\section{Retour à Reims, la pièce}

3 Dans sa version parisienne, Retour à Reims s'incarne sur scène par un trio d'acteurs/ trice: Irène Jacob (lookée chemise à carreaux-bottes en croco) y joue le rôle d'une comédienne engagée pour enregistrer la voix off d'un documentaire sur Didier Eribon, Cédric Eeckhout celui du réalisateur du film et Blade Mc Alimbaye, celui du propriétaire-rappeur du lieu où s'enregistre la session. L'action se déroule donc dans un studio d'enregistrement: une cabine de régie dans un coin, quelques meubles datés et une cafetière en arrière scène ; au centre du plateau, un pupitre encombré de câbles, de moniteurs, d'enceintes et un imposant micro. Au dessus, un très grand écran, pièce maitresse de l'agencement scénique ${ }^{6}$. Vont y défiler les images animées de Sébastien Dupouey et Thomas Ostermeier qui, dans un premier temps, suivent donc le « retour à Reims » d'Eribon, filmé en différentes situations "façon road trip ", non sans quelques clichés : seul dans le train, absorbé dans ses pensées, semblant inspiré par le paysage qui défile; dans des retrouvailles avec sa mère devant des photos familiales, un thé et des Petits Lu ; dans une librairie (il feuillette un livre de Derrida); dans un café avec son comparse Geoffroy de Lagasnerie; dans une archive de l'Ina (1989), sur le plateau d' Apostrophes, à l'occasion de la sortie de sa biographie sur Michel Foucault, etc. Les images défilent sans surprendre ni convaincre, mais sont toutefois rehaussées de longs passages du livre d'Eribon lus par Irène Jacob. Le dispositif imaginé peine à séduire tant le texte et «le théâtre » qui sont censés l'accompagner sont écrasés par le recours par 
trop pesant à ce documentaire naturaliste et surcodé dont on ne cesse de se dire qu'il s'agit là d'un artifice dont Ostermeier aurait pu (dû) se passer (glissement d'un théâtre documentaire à un « docuthéâtre " ?). L'écran animé censé faire le lien avec la "vérité " de l'ouvrage d'Eribon fait finalement barage au point d'ériger un de ces fameux "quatrièmes murs" disjoignant le public des comédiens. Et les moments dialogués, notamment entre Jacob et Eeckhout quant à la manière d'adapter Retour à Reims à l'écran n'y changent rien, tant ils s'avèrent peu probants, et ce, à tel point que le spectateur s'interroge, lui, plutôt, sur la façon dont l'ouvrage a été adapté à la scène.

4 À l'évidence, les choix tant scénographiques que dramaturgiques déçoivent. Ils se voient de surcroît affaiblis par de vrais ratés: faiblesse des dialogues; cabotinage; traits d'humour par trop appuyés; Mc Alimbaye qui décoche inopinément un rap toutefois bien envoyé, mais interpelle le public façon « chauffeur de salle »; usage d'un running gag prenant pour thème la préservation de la moquette du studio, etc. Tout le travail réflexif singulier, au cœur du travail d'Eribon se voit, in fine, traité comme vague source d'inspiration plutôt que comme matériau à interpréter. La réflexivité mise en scène est davantage celle des gens de théâtre qui s'interrogent assez maladroitement sur (le sens de) leurs pratiques: Eeckhout qui lance à Jacob, dans une mise en abîme grossière : "Détends-toi, on n'est pas au théâtre »; des images des publics de l'opéra dont le commentaire assène qu'ils "tirent une sorte de contentement de soi et un sentiment de supériorité » (p. 108 - qu'en est-il alors du public même de Retour à Reims ?); d'autres qui montrent la mobilisation des Gilets jaunes dont l'un des lieux de manifestation de prédilection n'est autre que le quartier des Champs Élysées où est implanté l'Espace Cardin... La démarche spéculaire en arrive finalement à poser, en creux, la question de la portée critique du théâtre, interrogation à laquelle Ostermeier va répondre en déployant, dans un second volet, une forme de théâtre qu'il estime précisément politique en ce qu'il s'adresse à la raison, là où son prédécesseur, Laurent Hatat, voyait plutôt la nécessité d'un "théâtre des idées ", mais ne pouvant passer que par l'émotion (un détour par les affects pour en arriver à la connaissance, selon le vœu de Brecht).

5 Ostermeier explique que l'adaptation l'a enjoint à « se demander comment faire de l'art engagé aujourd'hui. Est-ce qu'il ne faut pas être plus militant, arrêter de faire du théâtre et des films et descendre dans la rue avec les gens comme Sartre en 68 ? ». Et de poursuivre: «Toutes ces questions que je me pose, m'ont permis de traiter toutes les questions sur scène. C'est une obligation d'être très engagé car on vit des temps

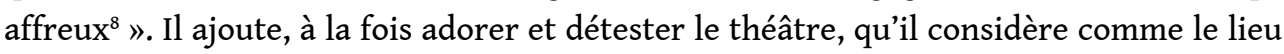
par excellence de la vanité, de la réalité illusoire, et espère avoir rendu son spectacle désagréable à la bourgeoisie qui viendrait le voir. Ostermeier oublie combien la réflexivité et la capacité à produire une distance à soi peut, certes, être au fondement d'une remise en cause de ses manières d'être au monde, mais être aussi configurée comme un dispositif de neutralisation de la critique. Important est en effet le risque de ce désarmement dans un théâtre où les publics et le dramaturge partagent notamment un ethos commun de classe et une connivence culturelle qui les constituent dans un Nous a priori inclusif. Le directeur de la Schaubühne déclare, par exemple, espérer provoquer des questionnements, notamment s'agissant des théories du complot ( $\mathrm{Je}$ sais que l'on va m'accuser de tomber dans une théorie du complot social» - p. 123), alors qu'à l'évidence, le capital culturel des spectateurs qui assistent à la pièce les prémunit plus sûrement que d'autres d'une adhésion naïve à ce type de pensée ; mais 
les expose peut-être davantage que d'autres aux tirs croisés et nourris du délire sondagier, des battages médiatiques et de certaines enquêtes sociologiques incomplètes ${ }^{9}$ qui participent à construire, volens nolens, les représentations négatives des mouvements sociaux. La justification, à ce titre, de la scène entre Irène Jacob et Cédric Eeckhout durant laquelle ils discutent de passages de l'ouvrage à conserver ou à retirer, au regard de la possibilité qu'ils puissent être assimilés à des éléments d'une théorie du complot, semble donc peu ajustée à la sociologie des publics qui en font réception. La «bonne » question, sur ce thème et au regard de la position sociale de la plupart des spectateurs de la pièce, ne devrait-elle pas porter davantage sur cette propension à penser qu'une large partie des Gilets jaunes (et plus largement des classes populaires) fut prise dans les rets du complotisme? Sur scène, les protagonistes finissent donc par se disputer, se séparent et quittent le studio... Fin du premier round. Cette première séquence semble finalement n'avoir été jouée que pour servir de warm up à la seconde partie du spectacle qui traiterait, elle, des « vrais » enjeux artistiques et $\mathrm{du}$ « véritable » sujet politique.

6 «Je me suis dit, qu'en tant qu'artiste, il fallait que je prenne position par rapport à la montée des populismes, et l'analyse de Didier Eribon explique beaucoup de choses sur la montée de l'extrême droite [c'est nous qui soulignons]. Alors j'ai rencontré Didier et j'ai commencé à imaginer une mise en scène de son texte Retour à Reims. Je pense que la force de ce livre c'est qu'il y a à la fois ce récit très intime, une sorte de confession et ensuite, à partir de ça, parce qu'il se pose des questions par rapport à sa famille et à son passé, il offre une analyse qui soutient qu'il y a un lien entre la corruption des élites de la gauche et la montée de l'extrême droite ${ }^{10}$.»

7 Les protagonistes reviennent donc, une semaine plus tard, pour une ultime session d'enregistrement de la voix off du documentaire, lequel a, entre-temps, épousé une nouvelle forme. Après réflexion, le réalisateur a décidé de ne plus mettre à l'écran Eribon, et son montage de faire finalement la part belle à des images censées illustrer une fois encore, plutôt maladroitement - la déchéance d'une gauche ayant abandonné les classes populaires, assumant son tournant libéral et favorisant la montée de l'extrême droite. Le phénomène est notamment symbolisé à l'écran par des vues de l'usine, aujourd'hui désaffectée, où la mère d'Eribon travaillait à la chaîne et dont les murs accueillent des affiches du Front National : les bastions ouvriers tenus par une CGT combative ne serviraient donc plus que de toile de fond à l'expression d'une droite nationaliste et raciste. Le travail à proprement parler d'auto-socioanalyse (mal) traité dans la première partie - notamment à distance de la réelle émotion que peut pourtant susciter la lecture de Retour à Reims - laisse alors place, à l'écran, à une suite d'archives filmées ordonnées chronologiquement (un rassemblement du Front populaire, un discours de Jacques Duclos, les événements de Mai 1968, l'élection de Mitterrand, le tournant Hollande, une mobilisation des Gilets jaunes, etc.) que l'on peine à envisager (parce qu'au théâtre et sous la direction de Ostermeier) autrement que comme une balourdise didactique au service d'une démonstration à l'emporte-pièce : d'une part, l'abandon de la classe ouvrière par la gauche de pouvoir européenne (Mitterrand, Schröder, Blair, Hollande) convertie depuis plus le début des années 1980 au libéralisme (fait avéré) et, d'autre part, et en conséquence, la conversion de la classe ouvrière aux thèses et aux votes les plus droitiers : les rouges seraient finalement devenus bruns (i.e. des «gaucho-lepénistes » pour parler comme Pascal Perrineau, politologue médiatique et garant du « Grand débat » macronien) : « Comment [sa famille] devint-elle une famille 
où il parut possible, et parfois presque aussi naturel, d'accorder son suffrage à l'extrême droite ou à la droite? » (p. 127).

8 À la scène, le tableau n'est guère plus intéressant. Le propos se pare des oripeaux du politiquement correct qui voudrait comme nuancer ce sur quoi Retour à Reims insiste par trop, un retour à la classe, et finalement de frayer avec le populisme. Aussi, Ostermeier aménage-t-il deux séquences particulièrement gauches: l'une censée illustrer la domination masculine et donc l'importance de la prise en compte des rapports sociaux de sexe (le réalisateur - petit mâle blanc imbu de sa personne confiant en cabine, mais tout micro ouvert : «C'est le gros cliché là ! C'est l'actrice qui croit qu'elle sait mieux que moi ce que c'est de faire un film »); l'autre supposée faire le pendant du côté des rapports sociaux de race : le propriétaire du studio, rappeur, noir, arborant une chemise en tissu africain (Ostermeier se défend d'en faire un stéréotype parce que le personnage est, affirme-t-il, « celui qui possède l'outil de production $»^{11}$ ), racontant le sort tragique réservé par l'État français à son grand-père, tirailleur durant la Seconde Guerre mondiale. Si l'on se place à l'endroit du théâtre, ces deux scènes n'ont hélas guère de sens. Ces tirades (particulièrement longue pour ce qui concerne la dernière) ne semblent devoir être là que pour satisfaire aux nécessités des paritésconcurrences victimaires et à celles d'une vulgate identitaro-intersectionnelle devenue passage obligé des discours critiques à la mode (buzzword) dont la tendance est de se transformer en bréviaires à partir desquels " tout le monde répète inlassablement la même chose, avec un tout petit répertoire d'idées ${ }^{12}$ ", pour finir en dogmes figés qui font paresseusement allégeance aux politiques des identités dont Eribon lui-même estime qu'elles font montre, à tout le moins en certains de leurs développements queer, d'une naïveté conduisant à croire « que le monde social n'existe pas et que les normes sont extérieures à ce que nous sommes et qu'il nous est loisible de les accepter ou de les refuser ${ }^{13}$ ». Ostermeier-Augias patauge alors dans les écuries poissées des bons sentiments et du politiquement correct ${ }^{14}$. L'adaptation que propose, là, le directeur de la Schaubühne semble s'être quelque peu perdue dans les méandres d'un théâtre qui se veut critique, mais qui, sans s'illusionner vraiment sur son efficace à proprement parler politique (il reconnaît que le politique se déploie dans les rues et non dans les salles de spectacle), semble cependant craindre d'être ringardisé à développer un point de vue de classe conséquent. Avant de revenir plus en détail sur ces concessions, commençons par faire retour sur Retour à Reims - l'ouvrage -, car c'est peut-être là que se trouvent les ferments de cette dramaturgie en berne.

\section{Retour à Reims, le livre}

9 Quel est le propos de Retour à Reims? Eribon en pose les bases de manière tout à fait explicite: son ouvrage porte sur la domination sociale, la possibilité qu'il a eue d'y échapper ( "J'avais fui ma famille [et sa classe d'origine] et n'éprouvais aucune envie de la retrouver »-p. 11), mais aussi sur la " personnalité dissociée » (la sienne) qui en est la conséquence. Contrairement à ce qui s'écrit parfois, Retour à Reims ne se veut pas être une simple autobiographie, mais plutôt une auto-socioanalyse - une enquête sociologique sur soi, « une analyse historique et théorique ancrée dans une expérience personnelle » (p. 22), une « introspection sociologique ${ }^{15} »-$ dont l'objectif est de rendre compte de la manière dont la prime socialisation de l'auteur a influencé son rapport au monde; non sur le mode de la servitude et de la conformation aux déterminismes, mais, a contrario et plus étonnamment, en échappant aux déterminations qui le 
prédestinaient, comme ses frères, à devoir s'inscrire dans un destin statistique qui est celui du milieu ouvrier dont il est issu (reproduction et non fatum). À l'instar de certains de ses illustres prédécesseurs ${ }^{16}$ - Richard Hoggart ${ }^{17}$ ou Pierre Bourdieu ${ }^{18}$-, Eribon va s'extirper du monde des classes populaires pour finalement devenir universitaire ${ }^{19}$ (d'abord aux États-Unis - Berkeley -, puis en France - Amiens) et occuper, de ce fait, une place plus enviable dans l'espace social. L'ouvrage revient donc sur sa trajectoire biographique et sa "qualité » de transfuge de classe échappant exceptionnellement au « verdict social» (résultat d'une délibération sociétale) auquel il était prédestiné, mais non sans être porteur d'un habitus clivé dont on n'apprend toutefois que peu de chose. Cette « dissidence » sociale prend racine très tôt et s'inscrit, au ras du sujet, dans des manières d'être au monde qui ne sont pas celles prévalant et attendues dans/de son environnement proche: divergences d'appétence (pour les études, la lecture, la culture), de (dé)goûts (e.g. le bricolage, la pêche vs. les livres, les arts) et d'orientation sexuelle (homosexualité) qui construisent peu à peu une sécession irrémédiable avec son milieu familial et nourrissent notamment la détestation d'un père-repoussoir qui incarne tout ce que le jeune Didier abhorre: «Je ne l'aimais pas. Je ne l'avais jamais aimé. [...] Rien ne nous attachait, ne nous attachait l'un à l'autre. [...] Il ne m'inspirait aucun sentiment, pas même de compassion. [...] Il m'avait engendré, je portais son nom, et, pour le reste, il ne comptait pas pour moi » (pp. 15-16-18). «J'ai avant tout détesté mon père parce qu'il était ouvrier ${ }^{20} »$.

10 Au cœur de ce travail réflexif, il est un sentiment qui traverse la biographie d'Eribon: celui de la honte - c'est-à-dire des choses qui se taisent et s'oublient, mais aussi de la honte d'avoir honte : «Je suis un produit de l'injure. Un fils de la honte »- qui participe du répertoire des affects minoritaires, au nombre desquels on trouve également la $p_{e u r^{21}}$. La honte d'être gay en premier lieu, qui sera au principe de ses principaux intérêts de connaissance et de nombreux de ses ouvrages, mais aussi la honte sociale dont il affirme qu'elle fut finalement plus ravageuse que sa prime honte sexuelle, dans la mesure où il fut impossible pour lui de construire quelque chose de valorisant à partir de cet héritage social-familial, notamment parce que la « terre d'asile » sociale et culturelle qui va l'accueillir (environnement intellectuel et gay) et à partir de laquelle il va enfin pouvoir se construire positivement (reconnaissance de ce qu'il est et fait) ne sollicite rien du legs dispositionnel de son enfance-adolescence : « un espace social que j'avais mis à distance, un espace mental contre lequel je m'étais construit, mais qui n'en constituait pas moins une part essentielle de mon être » (p. 13). Cette terre d'exil serait même traversée par un ethnocentrisme (mépris) de classe qui ne dit pas toujours son nom, mais opère efficacement, l'incitant à refouler encore davantage son prime habitus de classe : « une origine que j'avais du mal à assumer dans les nouveaux milieux dans lesquels j'évoluais ${ }^{22} »$. Et de s'interroger :

11 «Pourquoi moi, qui ai tant éprouvé la honte sociale, la honte du milieu dont je venais quand, une fois installé à Paris, j'ai connu des gens qui venaient de milieux sociaux si différents du mien, à qui souvent je mentais plus ou moins sur mes origines de classe, ou devant lesquels je me sentais profondément gêné d'avoir ces origines, pourquoi n'aije jamais eu l'idée d'aborder ce problème dans un livre ou un article?» (p. 21).

12 Retour à Reims pose donc à nouveaux frais empiriques ${ }^{23}$, c'est-à-dire dans le cadre de la singularité plurielle de son auteur, la question de la violence symbolique qui s'exerce, en l'espèce, à deux niveaux : à un premier niveau, celle qui situe Eribon dans une tension permanente entre des prescriptions de classe qui, notamment par le biais familial, lui 
dictent des comportements censés convenir, mais auxquels ils n'adhèrent pas et, précisément, une personnalité désajustée, non conforme aux attendus de son milieu d'origine, laquelle lui permettra précisément de se dégager de celui-ci; à un second niveau, celle qui l'intimera de se départir de toute relation avec ses origines sociales et de faire comme s'il n'était pas un transclasse ${ }^{24}$ nécessairement chargé de traces de ses origines et restant soumis aux effets du passé et des socialisations antérieures : «Ce à quoi l'on a été arraché ou ce à quoi l'on a voulu s'arracher continue d'être partie intégrante de ce que l'on est " (p. 14). Retour à Reims s'attache ainsi à montrer la force de ces assignation divergentes, clivantes, qui enjoignent, chacune à leur manière et depuis des valeurs et des dignités propres, à différer d'un territoire social comme condition à se réengager dans un autre et pouvoir, in fine, se sentir " être des nôtres » : «Au fond, j'étais marqué par deux verdicts sociaux : un verdict de classe et un verdict sexuel. On n'échappe jamais aux sentences ainsi rendues» (p. 230). Ces formes de violence symbolique enchâssées conduisent, sur un plan individuel, à des phénomènes de double dissociation alimentés par des processus coûteux de désidentificationréidentification :

« Tenir les deux sphères ensemble, appartenir sans heurts à ces deux mondes [univers scolaire et familial] n'était guère possible. Pendant plusieurs années, il me fallut passer d'un registre à l'autre, d'un univers à l'autre, mais cet écartèlement entre les deux personnes que j'étais, entre les deux rôles que je devais jouer, entre mes deux identités sociales, de moins en moins liées l'une à l'autre, de moins en moins compatibles entre elles, produisait en moi une tension bien difficile à supporter et, en tout cas, fort déstabilisante » (p. 171).

«Et je porte en moi la marque de l'un et de l'autre. Mais parce qu'ils entrèrent en conflit l'un avec l'autre à un moment de ma vie, je dus me façonner moi-même en jouant de l'un contre l'autre » (p. 230).

La lecture de Retour à Reims, ne nous renseigne toutefois, répétons-le, que peu sur ce que son auteur a malgré tout conservé de sa socialisation primaire. Si Eribon décrit fort bien son statut de transfuge de classe, très peu de détails nous sont livrés quant à ce qu'il conserve comme trace active de son passé. Ce manque dit en creux ce qu'il en coûte à l'auteur d'avoir à se désaligner, ne serait-ce que momentanément, d'avec sa classe de ralliement. Il témoigne de la force des réaffiliations - qui sont de facto aussi des désaffiliations - qui enjoignent de lever les amarres des territoires barbaresques pour rejoindre des côtes plus civilisées et de s'y installer définitivement. Tout semble se passer comme si son intégration à l'environnement électif dont il est aujourd'hui membre (espace social d'arrivée) avait été total et les phénomènes d'hystérésis liés aux dispositions acquises quasi absents, si ce n'est dans ce sentiment de contrition d'avoir honte d'avoir (eu) honte, révélant ainsi, malgré lui et contre toute attente, un attachement affectif à son milieu d'origine, une sorte de lien invisible le connectant encore à ce dernier. Eribon en a par ailleurs conscience et de s'interroger: "Toute entreprise autobiographique et même toute entreprise autoanalytique est amenée, à un moment ou à un autre, à s'interroger sur l'atavisme des qualités et des défauts, des traits physiques et des traits de caractère. Mais que reste-t-il des ces ressemblances, chez un transfuge de classe, quand tant de dissemblances s'installent et cherchent à acquérir la suprématie sur elles ? $^{25} »$. Plus exactement, sa construction comme jeune intellectuel gay contrevenant "aux lois de la normalité sexuelle» semble l'avoir conduit à scotomiser ce qui en lui pourrait relever du monde ouvrier (Eribon parle à cet 
égard de "rééducation »), lequel a pourtant constitué, à un moment de sa vie, l'essentiel de son entourage. Ce refoulement individuel (une subjectivation en résistance) maintenant à distance des parcelles de soi éprouvées comme peu conciliables avec un Moi «nouveau " épouse également les atours d'une sorte de forclusion sociale par pression du milieu d'adoption, lequel semble l'encourager à s'arranger pour que son milieu d'origine ne puisse exercer son "droit de suite", notamment par le maintien d'une distance irrémédiable avec la famille et « en laissant de côté tout ce qui en [lui], dans [sa] propre existence, aurait pu, aurait dû, [le] conduire à orienter aussi [son] regard sur les rapports de classe, la domination de classe» (p. 23). Le regard d'Eribon est, sous cet angle, tributaire du point de vue de la classe qui est aujourd'hui le sien, une vue informée par des schèmes de perception qui sont ceux d'un groupe social dominant. De facto, on n'apprend que peu de choses du réagencement pratique de ses schèmes dispositionnels, du volontarisme dont il a nécessairement fait montre pour se reprogrammer et des « techniques de soi » qui s'y rapportent. On pourrait avancer, qu'ici, Eribon n'est ni assez bourdieusien, ni assez foucaldien, ni même assez sociologue.

Le cas de sa socialisation politique dans les rangs d'une organisation trotskiste n'est ainsi jamais appréhendée en termes de ressources l'ayant aidé à pénétrer l'espace social visé26 (et pourtant!), mais comme un appui lui ayant permis, à certains égards, de mettre encore davantage sa famille à distance et de renforcer sa désidentification sociale (parents affidés au PCF, mais bassement matérialistes, « embourgeoisés » : «Et si j'étais marxiste, je dois avouer que le marxisme auquel j'adhérais pendant mes années d'études, comme mon engagement gauchiste, n'étaient peut-être qu'une façon d'idéaliser la classe ouvrière, de la transformer en une entité mythique en regard de laquelle la vie de mes parents m'apparaissait bien condamnable »- p. 86), tout en étant le lieu d'expériences négatives ("J'étais coupé en deux: moitié trotskiste, moitié gay. Deux identités séparées, qui semblaient inconciliables »-p. 205). On a, là, l'impression que Eribon a dû « choisir son camp » critique, comme si, le fait d'affirmer une identité d'intellectuel par ailleurs gay devait nécessairement fermer les portes d'une attention à la domination sociale de type classiste, "redoublant peut-être par le geste de l'écriture théorique ce qui avait été la trahison existentielle»(p. 28). Dans La société comme verdict, Eribon continuera à examiner son transclassime en soulignant la grande difficulté qu'il ressent à ne plus éprouver cette honte qu'il a pourtant objectivée en la décortiquant, rappelant ainsi que l'émancipation "par/de l'esprit » (une conscience volontaire) ne recouvre pas l'émancipation "par/de corps »: "Il ne suffit pas de prendre conscience de la violence que l'ordre social exerce sur les individus, [...] il ne suffit même pas de l'analyser, pour être en mesure de n'y être plus assujetti, et de ne plus s'y soumettre ${ }^{27} »$. Mais de cela, Ostermeier n'aura rien voulu/pu retenir...

\section{La critique, c'est (la) classe !}

17 Le responsable de la Schaubühne estime qu'une pièce ne vaut que pas son étrangeté, par le fait qu'elle "convoque l'inconnu ${ }^{28}$ ». Est-ce à dire que son intérêt pour Retour à Reims fut également motivé par un texte qui révèlerait quelque bizarrerie ? S'il est à parier que l'une de ces surprises fut de se retrouver pour partie dans le témoignage d'Eribon ("Quand j'ai fermé le livre, je me suis dit: “tiens, au moins, tu n'es pas tout seul” " - la projection spéculaire plus ou moins déformée est un ressort évident de l'attachement aux œuvres), une autre semble être liée à l'importance des questions de 
classe : « Il y a un retour de la lutte des classes, mais il faut penser les classes de manière différente" (D. Eribon sur France Culture, janvier 2019). Retour à Reims serait-il un retour à la classe, voire à la lutte des classes ? Auquel cas, dans quel gouffre auraientelles préalablement chu avant d'en être sorties ? Le lieu de leur supposée disparition ${ }^{29}$ ne se résume-t-il pas à l'esprit de certains pour qui la « question sociale» doit s'éclipser pour mieux laisser la place à l'importance d'autres oppressions ? Eribon oscille en fait entre deux explications : d'une part, la disparition pratique de la classe ouvrière en tant que classe consciente d'elle-même, mobilisée (absence pure et simple du référent); d'autre part, le rejet théorique de la classe par des formes de critique "censurant ou refoulant ce que le marxisme nous avait accoutumés à "percevoir" comme l'unique forme de domination» (p. 244 - absence des mots). Il avance ainsi que les thèmes identitaires des «nouveaux mouvements sociaux" "offraient des catégories pour se penser soi-même, et donc pour penser [et panser] son enfance et son passée ${ }^{30}$ ». Mais la perspective classiste ne permet-elle pas d'accomplir une telle opération? À l'évidence, la réponse est positive et l'explication du choix qu'opère Eribon tient moins à la faiblesse heuristique du marxisme à penser l'individu qu'à la difficulté de coupler des approches valorisant une singularité plurielle irréductible du sujet avec celles qui considèrent - non pas a contrario, mais alter apud alternam - que le particulier relève aussi de logiques sociales englobantes qui, si elles n'empêchent pas la diversification des trajectoires biographiques et des ethos, en cadrent néanmoins le développement. Il faudrait d'ailleurs considérer d'un peu plus près en quoi ce qu'Eribon dénonce s'agissant du tournant idéologique de la gauche de gouvernement («l'exaltation du "sujet autonome" et la volonté concomitante d'en finir avec les pensées qui s'attachaient à prendre en considération des déterminismes historiques et sociaux " p. 131), ne se retrouve pas aussi, pour partie, au principe de modèles théoriques, à l'instar des Identity Politics, qui ont tôt fait de décréter que la classe serait de peu de valeur pour penser les antagonismes et, de surcroît, un concept à tendance égocidaire. Mais « prétendre que l'extrême singulier serait l'endroit du politique, [ne serait-ce pas] diluer un peu plus le politique dans le marché $?^{31} »$. Surtout, les armes des mille marxismes permettent une critique de la (petite-)bourgeoisie, mais s'y plier réflexivement depuis ce cadre théorique semble devoir par trop ressembler, pour Eribon, à la possibilité de détruire partiellement la piste d'atterrissage qui lui a précisément permis d'envisager son envol. L'éventualité de cette trahison - cette fois vis-à-vis de sa classe d'adoption - lui est sans doute insupportable tant elle invaliderait un parcours de vie arraché de haute lutte, marqué par la souffrance et la honte. On ne ruine pas tant d'efforts par choix théorique: "Montrer ce que l'on est devenu est agréable et valorisant. Montrer ce qu'on était auparavant l'est beaucoup moins ${ }^{32}$ ». Montrer en quoi ce désaveu des origines sociales est aussi un jugement de classe serait semble-t-il insoutenable. La chose n'échappe d'ailleurs pas à l'universitaire amiénois qui concède que «le dépassement du reniement initial ne peut prendre la forme d'un reniement de ce qui l'a déterminé, c'est-à-dire de toutes les ressources qu'offre la culture dominante ${ }^{33} »$.

18 Par ailleurs, à plusieurs reprises, Eribon semble se laisser prendre au travers métonymique (plus précisément synecdochique) : sa famille vaut pour les milieux populaires, la section du groupe trotskiste dans lequel il militait pour les divers marxismes, l'intérêt « actuel » pour les approches classistes pour un retour à « un vieux marxisme stérilisant », le racisme de certains ouvriers pour une maladie chronique des classes populaires ${ }^{34}$, les penchants homophobes de certains «lacanismes» qui 
envisagent l'homosexualité comme un développement morbide pour toute la psychanalyse, etc. :

19 "J'étais politiquement du côté des ouvriers, mais je détestais mon ancrage dans leur monde. Me situer dans le camp du "peuple" eût sans doute suscité en moi moins de tourments intérieurs et de crises morales si le peuple n'avait pas été ma famille» (p. 73).

20 "La captation par l'extrême droite de l'ancien électorat communiste [...] fut rendue possible ou facilitée par le racisme profond qui constituait l'une des caractéristiques dominantes des milieux ouvriers et populaires blancs » (p. 146).

21 Cette tendance à prendre la partie pour le tout se convertit, en certains développements, en des formes de réification particulièrement fustigeantes, par exemple quand il évoque les «sentiments haineux et notamment racistes qui préexistaient chez eux [ses parents parangons de la classe ouvrière] depuis toujours [c'est nous qui soulignons] $]^{35} »$; la violence domestique comme " un trait caractéristique de la vie des couples hétérosexuels durables - des couples ouvriers, du moins ${ }^{36} »$; ou encore le fait qu'il n'y aurait pas de mémoire familiale ouvrière. La classe ouvrière semble ainsi, parfois, figée dans ses impasses et incapables de s'élever de sa condition, réduite à une classe (im) probable à mille lieues d'un prolétariat organisé et combatif (sujet de l'émancipation), cherchant « à faire construire un pavillon » plutôt qu'à « faire la révolution ${ }^{37}$ et finissant même par voter pour le Front national. La vision d'Eribon est, sur ce point, finalement assez peu éloigné du trop connu rapport du think tank Terra Nova - Gauche : quelle majorité électorale pour 2012? - qui justifie l'abandon par le Parti Socialiste des classes laborieuses, par le fait que le FN serait devenu le nouveau parti refuge des ouvriers, et que travailler au retour à gauche des «beaufs" serait une peine bien inutile et électoralement peu rémunératrice, contrairement à l'effort à fournir vis-à-vis des populations les plus diplômées, des jeunes urbains, des femmes, des «minorités » et de "la France de la diversité ». Pour Gérard Mauger, cette «stigmatisation du peuple mal votant [...] renoue - peut-être sans le vouloir et sans le savoir - avec la philosophie conservatrice de la fin du XIX ${ }^{\mathrm{e}}$ siècle et sa méfiance des foules et de la démocratie ${ }^{38}$ ». Les récents commentaires fielleux ${ }^{39}$ sur le mouvement des Gilets jaunes, proférés à l'encan par le Président Macron, les membres du gouvernement, nombre d'élus (députés, sénateurs, maires), les éditocrates patentés et quelques intellectuels médiatiques impavides, tendent évidemment à renouveler ce profond dédain pour le peuple dont la mobilisation relèverait nécessairement d'un populisme $e^{40}$ rétrograde (xénophobe, homophobe, antisémite, etc.), qualification dépréciative «qui place d'emblée sous un signe négatif toutes les mobilisations populaires ${ }^{41} »$.

22 Les enquêtes sérieuses de sociologie électorale dressent pourtant un portait du vote ouvrier nettement plus mesuré. Si l'influence du FN «a grandi régulièrement, de manière cachée et rampante ${ }^{42} »$, elle n'a, pour autant, jamais été majoritaire, contrairement à l'abstention ${ }^{43}$ (laquelle est considérée par d'aucuns comme un privilège de blancs ${ }^{44}$ ), surtout dans les rangs des ouvriers qui votaient habituellement à gauche : « il est impossible de prédire, à coup sûr, quels sont les ouvriers qui vont voter Le Pen et quels sont les ouvriers qui ne vont pas voter Le $\operatorname{Pen}^{45}$ ». " Certes, nous avons trouvé des ouvriers qui avaient voté communiste et qui se sont mis, par moments, selon le contexte, à voter $\mathrm{FN}$, mais très peu de militants de gauche qui soient passés durablement de l'autre côté46 $»$. Malgré ces nuances, l'idée d'une convergence culturelle 
globale des milieux ouvriers avec les valeurs de l'extrême droite - laquelle se traduirait électoralement par des votes d'adhésion - semble constituer un « doxème " difficile à déconstruire, dont les manifestations liminaires remontent au mitan des années 1980. Si le racisme et la " préférence nationale » existent de facto dans les rangs ouvriers, ils ne sont pas hégémoniques et ne signent pas une conversion intégrale des classes populaires à l'idéologie du FN, tant s'en faut. Stéphane Beaud et Michel Pialoux, soulignent, à cet égard, combien ce qu'ils nomment les " pulsions racistes ", quand elles existent, sont par ailleurs souvent contrebalancées par le sentiment "d'une même condition sociale faite d'"exploitation" au travail, [...] d'un destin collectif de classe, d'une nécessité de "défendre" les mêmes droits en tant que travailleurs ${ }^{47}$ ", c'est-à-dire par l'instinct de partager un destin de classe analogue, dont la prise conscience peut constituer, à tout le moins, le socle de revendications et de combats communs.

Pour Eribon, force est de constater que si charge rébellionnaire il y a, celle-ci se niche plutôt dans "l'exploration des strates de la honte» et, plus précisément, dans la création de nouvelles significations sociales liées à l'analyse des « formes incorporées de l'infériorisation ${ }^{48}$ ». De facto, dans le triptyque Retour à Reims, il s'intéresse surtout à la dimension culturelle des antagonismes sociaux et aux catégories symboliques qui s'y rapportent (e.g. au travers de ses piquantes analyses littéraires). Aussi, parle-t-il et juget-il les classes populaires, et les discours qu'il tient sur elles sont traversés de contradictions patentes, preuves de la vision tremblée et des sentiments clivés qu'il développe malgré lui à leur égard ${ }^{49}$. Les représentations qu'il en propose sont donc "pendulaires ", mais finissent plutôt par balancer du côté d'un " "populaire négatif" (supposé particulièrement réceptif aux sirènes du Front national), [passant] de la célébration de "l'homme de marbre" à la stigmatisation du "beauf" : inversion du signe corollaire de la disqualification du marxisme au cours des années $1970^{50}$ ». Et son doctorant Édouard Louis, romancier à succès lui aussi adapté par Ostermeier (et, récemment, par Stanislas Nordey), de souligner dans un billet transmis à la revue Les Inrockuptibles : « Pour les dominants, les classes populaires représentent la classe-objet par excellence, pour reprendre l'expression de Pierre Bourdieu ; objet manipulable du discours : de bons pauvres authentiques un jour, des racistes et des homophobes le lendemain ${ }^{51} »$. On ne saurait mieux résumer.

\section{Conclusion}

En adaptant Retour à Reims, Thomas Ostermeier affirme vouloir faire un théâtre politique qui fait réfléchir, notamment à l'arrière-fond social de ce qui est mis en scène. Il s'agit alors, dans une veine brechtienne, de se dépouiller des oripeaux d'une dramaturgie du faire-semblant et de l'illusion au profit d'un théâtre qui est plutôt censé expliquer. Aussi, Irène Jacob, Cédric Eeckhout et Blade Mc Alimbaye y incarnent moins des personnages avec une épaisseur singulière propre, que des "raconteurs", jouant " entre guillemets ${ }^{52}$ ", à distance. Ce choix - compréhensible au égard au principe brechtien du verfremdungseffekt - est somme toute étrange dans la mesure où il prend le contre-pied de l'« intimisme » qui fait l'une des spécificités de l'ouvrage d'Eribon et pour ne valoriser de ce dernier que sa partie la moins intéressante : celle qui voudrait tirer des leçons et des conclusions d'un diagnostic du présent dont nous avons vu qu'il était quelque peu hâtif et abusif. Si Ostermeier se garde pour autant de reprendre le " refrain » populaire-populiste, il n'ose, cependant, aller au bout de la logique d'un théâtre qui se voudrait dialectique, insistant franchement sur les affrontements de 
classe. Le «trouble » qui traverse la pièce relève en fait d'une triple nature. D'une part, il ressortit donc du décalage entre les ascendances d'un théâtre politique et le caractère le plus notoire de l'œuvre mise en scène qui ne n'est pas facile à faire rentrer dans les canons de l'épique; d'autre part, la gêne vient également du fait que la portion la plus directement politique de Retour à Reims (le livre) s'avère la plus faible analytiquement, mais que c'est pourtant cet aspect que Ostermeier choisit finalement d'accentuer; embarras enfin, parce qu'il ne croît pas au contrat spectatoriel que suppose cette option (conscientisation) et donc à la possibilité de réarmer les facultés critiques de son auditoire. Cachez ces contradictions que la « bourgeoisie culturelle » ne saurait voir ${ }^{53}$ !

Le thème des antagonismes de classe traverse le livre, la pièce et ses publics, mais Ostermeier semble vouloir le pondérer par des questions identitaires qui, pour autant qu'elles sont d'importance, n'apparaissent, en l'espèce, convenir qu'en ce qu'elles rendent la lumière portée sur les affrontements de classe moins crue. C'est là un choix qui nous semble jouer sur la coïncidence de l'individualisme (petit-)bourgeois ${ }^{54}$ résonnant (raisonnant) avec les irréductibilités identitaristes, s'accordant pour désigner et condamner ce(ux) qu'ils considèrent être des ennemis communs, au nombre desquels les approches marxistes, la critique classiste, ainsi que les individus qui s'en réclament, vite dénoncées comme d'immondes totalitaires. Contrairement au meilleur de ce qui se joue au sein des théâtres «identitaires " et " de l'immigration » qui parviennent à affirmer "des motifs de fierté - en riposte à la honte ${ }^{55}$ ", en s'appuyant sur des expériences oppressives intimes, mais en les recontextualisant dans une totalité, Ostermeier ne réussit pas à dé-sérialiser les témoignages livrés par les comédiens malgré ses efforts de distanciation. À la scène, les personnages ne semblent guère esquisser un Nous, pas plus que ne semble pouvoir se créer de "concrétion " citoyenne - ne serait-ce que probable - avec la salle. Et à l'instar de Denis Guénoun cité par Olivier Neveux de s'interroger : « quelle est cette assemblée qui s'assemble, quel est ce nous ici même réuni? Qui est ce nous? Qui sommes-nous? Qui nous assemble? Que faisons-nous ensemble $?^{56} "$, ou à tout le moins, que pourrions-nous faire ensemble ? Telle que donnée en représentation, Retour à Reims n'offre pas une image du monde "propre à favoriser la pratique " (Brecht). La pièce n'engage à/en rien. Elle ne fédère pas, ne dessine aucune convergence, n'articule pas d'intérêts communs; elle se contente de juxtaposer des récits de vécus, orphelins d'un cadre collectif qui permettrait de les appréhender comme différentes facettes d'un même phénomène.

Il s'agit là d'une posture qui nous semble à la fois timorée - en ce qu'elle n'ose aller au bout de ses prétendues « convictions » - et par trop dans un air du temps critique qui tend à faire de la reconnaissance des identités (souvent figées, parfois essentialisées) et de la diversité (dont les premiers profits sont tirés par le marché), les nouveaux jalons d'une émancipation libéralo-compatible; repères paradoxaux dans la mesure où ils en appellent à une autonomisation des subjectivités résistantes et des sphères de la conflictualité sociale (à chacun sa m... lutte ${ }^{57}$ ), tout en semblant parfois se satisfaire de possibilités nouvelles d'inclusion sociale (et des réformes d'un "homo politicus ambidextre $^{58}$ » dont Emmanuel Macron est aujourd'hui la parfaite incarnation). Dans cette perspective - qui ne semble pas être celle de Ostermeier - la nécessité du dépassement des rapports sociaux de classe est dissoute dans les eaux troubles d'un egotisme agonistique (virant à la concurrence victimaire) pour lequel les politiques du conflit se transforment en lutte des places, ainsi qu'en chasse aux «privilèges » et aux micro-violences faisant éventuellement système. L'exigence de la construction d'une 
contre-hégémonie conséquente en vient à se résumer au fait de devoir s'allier compassionnellement aux victimes qui "savent » et dont on ne saurait discuter ni les souffrances, ni les conséquences politiques qu'elles en tirent. Ostermeier ne se fourvoie pas dans ces fausses évidences, mais peine cependant à mettre en énigme la réalité sociale qu'il entend représenter. En adaptant Retour à Reims, il a tenté une pièce à conviction, mais celle-ci semble ne pouvoir être produite qu'à la charge d'un certain « populaire » et de la critique classiste qui s'y rapporte. Sans doute s'agit-il moins d'une prise de position courue et assumée par le dramaturge allemand que la conséquence d'une difficulté conjoncturelle à ne pas se trouver affecté par un postmodernisme qui préfère définitivement les singularités martyrisées à la classe mobilisée et les trajectoires individuels aux destins collectifs.

\section{NOTES}

1. Dès sa sortie en Allemagne, Rückkehr nach Reims a été un incontestable succès de librairie (80 000 exemplaires écoulés en quelques mois). L'ouvrage a été beaucoup commenté dans les médias, ainsi que dans les milieux militants. La Fondation Rosa Luxembourg y a, par exemple, consacré une brochure : Klasse, scham und die linken. Debatte zu Eribons Rückkeher nach Reims (https://www.zeitschrift-luxemburg.de/debatte-zu-eribons-rueckkehr-nach-reims/).

2. Une adaptation avait déjà été proposée en 2013 par Laurent Hatat à l'espace Michel Simon de Noisy-le-Grand (https://www.theatre-contemporain.net/spectacles/Retour-a-Reims) puis à Avignon. Par ailleurs, Ostermeier a adapté, en 2018, à la Schaubühne (en coproduction avec le Théâtre de la ville), Histoire de la violence d'Édouard Louis, dont l'ouvrage En finir avec Eddy Bellegueule (Paris, Seuil, 2014) peut être envisagé comme le pendant littéraire de Retour à Reims, lui aussi inspiré par Pierre Bourdieu et Annie Ernaux.

3. https://www.theatredelaville-paris.com/files/e2e6d79e/retour_a_reims_pedago.pdf.

4. Ibid.

5. Il défend, de facto, une conception du théâtre « qui ne le rédui[t] pas à un espace de création artistique, mais défen[d] également sa place comme un espace de pensée dans la société, dans la cité. Le théâtre doit pouvoir traduire un point de vue sur la société. Il a une responsabilité politique " (Ostermeier in Chalaye (Sylvie), Thomas Ostermeier, Arles/Paris, Actes Sud/CNSAD, 2006, p 34). Ostermeier revendique de s'inscrire, en cela, dans la longue tradition du théâtre politique (Meyerhold, Piscator, Brecht, Weiss, etc.).

6. Vsevolod Meyerhold et Erwin Piscator ont, dès les années 1920, mêlé les images animées à leurs mises en scène. Cf. Piscator (Erwin), Le Théâtre politique, Paris, L'Arche, 1962.

7. Les références qui suivent et qui n'indiquent seulement qu'un numéro de page sont toutes tirées de Eribon (Didier), Retour à Reims ? Paris, Flammarion, 2010.

8. Ostermeier (Thomas), « Entretien avec Marie Richeux », Les temps qui courent, France Culture, 25 janvier 2019, https://www.franceculture.fr/emissions/par-les-temps-qui-courent/thomasostermeier.

9. Cf. par exemple : https://www.conspiracywatch.info/enquete-complotisme-2019-focus-sur-lemouvement-des-gilets-jaunes.html ; https://www.franceculture.fr/sociologie/gilets-jaunes-ceque-la-toute-premiere-enquete-raconte-de-la-revolte.

10. Ibid. 
11. Ibid. La dés-assignation passerait donc par l'entrepreneuriat.

12. Eribon (Didier), Retours sur Retour à Reims, Paris, Éditions Cartouche, 2011, p. 64.

13. Ibid., p. 65.

14. Barbéris (Isabelle), L'art du politiquement correct. Sur le nouvel académisme anticulturel, Paris, PUF, 2019.

15. Eribon (Didier), La société comme verdict. Classes, identités, trajectoires, Paris, Flammarion, 2014, p. 11 .

16. Dont il fait d'ailleurs une copieuse critique dans La société comme verdict (2014).

17. Hoggart (Richard), 33 Newport Street. Autobiographie d'un intellectuel issu des classes populaires anglaises, Paris Seuil, 2013.

18. Bourdieu (Pierre), Esquisse pour une auto-analyse, Paris, Raisons d'agir, 2004.

19. Il occupera auparavant les fonctions de journaliste littéraire (Libération, Le Nouvel Observateur).

20. Eribon (Didier), Retours sur Retour à Reims, op. cit., p. 22.

21. Cf. Eribon (Didier), Réflexions sur la question gay, Paris, Flammarion, 2012.

22. Eribon (Didier), « Entretien avec Sylvain Bourmeau », La suite dans les idées, France Culture, 10 octobre 2009, https://www.franceculture.fr/emissions/la-suite-dans-les-idees/retour-reims-ouesquisse-dune-autosocioanalyse-avec-didier-eribon.

23. Empirique davantage que théorique, dans la mesure où l'appareillage conceptuel mobilisé est largement emprunté à Pierre Bourdieu et à son Esquisse pour une auto-analyse (2004). Par ailleurs, il faut noter que l'écriture d'Eribon est plus littéraire que celle de Bourdieu et, ainsi, s'avère peutêtre plus à même de rendre sensiblement compte des violences et des souffrances qu'elle décrit et analyse.

24. Cf. Jaquet (Chantal), Les transclasses ou la non-reproduction, Paris, PUF, 2014.

25. Eribon (Didier), La société comme verdict..., op.cit., p. 31.

26. Eribon reproche au travail auto-socioanalytique de Pierre Bourdieu de dire trop peu : «Il tait plus de choses qu'il n'en confesse. [...] Il ne mentionne aucun des livres qu'il lisait, ne donne aucun renseignement sur ceux qui comptèrent pour lui ou lui donnèrent le goût de la culture, de la pensée... » (pp. 165-166). Non sans culot, il avance que dans Retour à Reims, il part de lui «pour aller vers l'analyse des déterminismes sociaux, tandis que Bourdieu dans Esquisse " n'évoque les déterminismes sociaux que pour aller vers lui même [en] "monarque absolu" " (La société comme verdict..., op.cit., pp. 72-73). Mais lui-même reste silencieux sur nombre d'éléments dont on aurait pu penser qu'ils furent pourtant des plus importants (cf. infra). Sans doute est-ce d'ailleurs, là, une limite de l'exercice auto-réflexif, toujours sur le fil de la retenue-censure et de l'illusion biographique.

27. Eribon (Didier), La société comme verdict..., op.cit., p. 36-37.

28. In Chalaye (Sylvie), op. cit., p. 34.

29. Stéphane Beaud établit un diagnostic similaire au sein même de la sociologie au sein de laquelle « un certain nombre d'objets, parmi les plus anciens et les plus légitimes de la sociologie, sont aujourd'hui peu traités ou tout simplement oubliés [et] c'est peut-être dans le domaine de l'analyse des classes sociales et de la stratification sociale que les manques, tant en termes de données statistiques qu'en termes d'enquêtes de terrain, sont devenus les plus criants de telle sorte qu'on assiste, en ce domaine, à une véritable régression sur le simple plan des connaissances »: Beaud, Stéphane, "La sociologie française au milieu du gué », SociologieS, 2012, http://journals.openedition.org/sociologies/3880.

30. Eribon (Didier), Retours sur Retour à Reims, op. cit., p. 14.

31. Barbéris (Isabelle), L'art du politiquement correct..., op. cit., p. 71.

32. Eribon (Didier), La société comme verdict..., op.cit., p. 20.

33. Ibid., p. 86.

34. Dans un entretien donné à Hérodote ( $\left.\mathrm{n}^{\circ} 162,2016\right)$, Patrick Braouezec, ancien maire de SaintDenis, actuellement Président de Plaine Commune et réencarté depuis 2017 au PCF - après avoir 
été un soutien d'Emmanuel Macron aux élections présidentielles afin, affirmait-il alors, de faire barrage à l'extrême droite, dès le premier tour -, avançait: " dans la classe ouvrière, il y a toujours eu une ambiguïté, du non-dit, vis-à-vis de l'étranger ».

35. Eribon (Didier), La société comme verdict..., op.cit., p. 52.

36. Ibid., p. 135.

37. Ibid., p. 191. Eribon pratique, là, ce qu'il reproche précisément au travail de Richard Hoggart, à savoir : manquer de discernement et faire fi de la complexité des dispositions populaires, car, rappelle-t-il, il s'agit de convenir qu'il y a « des différenciations internes à un milieu, à un groupe social, et $a$ fortiori à une classe sociale » (Ibid., p. 205).

38. Mauger (Gérard), « Mythologies. Le "beauf" et le "bobo" », Lignes, $n^{\circ}$ 45, 2014, p. 136.

39. Cf. https://www.acrimed.org/-Mobilisations-des-gilets-jaunes-2018-.

40. Terme dont Annie Collovald rappelle qu'il était utilisé, au début des années 1980, pour désigner la «nouvelle droite » américaine et, en France, le FN.

41. Collovald (Annie), «Le populisme: de la valorisation à la stigmatisation du populaire», Hermès, $n^{\circ} 42,2005$, pp. 157.

42. Beaud (Stéphane), Pialoux (Michel), «Pourquoi la gauche a-t-elle perdu les classes populaires ?", Savoir/Agir, $n^{\circ} 34,2015$, p. 65.

43. «Contrairement à une thèse désormais bien ancrée, le FN n'est donc pas le choix majoritaire des ouvriers et seule une petite minorité d'entre eux (moins de un sur 7) a pu céder sur les derniers scrutins à la tentation frontiste, soit en réintégrant dans l'analyse les non inscrits et les abstentionnistes, une propension finalement moindre que celle des indépendants " (Lehingue (Patrick), " "L'électorat" du front National. Retour sur deux ou trois "idées reçues" ", in Mauger (Gérard), Pelletier (Willy), coord., Les classes populaires et le FN. Explication de votes, Vulaines-surSeine, Éditions du Croquant, 2016, p. 37. Cf. également : Braconnier (Cécile), Mayer (Nonna), Les inaudibles. Sociologie politique des précaires, Paris, Presses de Sciences Po, 2015.

44. https://lavamedia.be/fr/sabstenir-est-il-un-privilege-de-blanc/. Et de penser que voter Macron pourrait être un privilège de riche!

45. Gougou (Florent), « Les ouvriers et le vote Front national. Les logiques d'un réalignement électoral ", in Crépon (Sylvain) et al., dir., Les faux-semblants du Front national, Paris, Presses de Sciences Po, 2015, p. 339.

46. Beaud, Stéphane, Pialoux (Michel), «Racisme ouvrier ou mépris de classe? Retour sur une enquête de terrain ", in Fassin (Éric) et al., dir., De la question sociale à la question raciale ?, Paris, La Découverte, 2006, p. 86. Cf. également: Mischi (Julian), «Essor du FN et décomposition de la gauche en milieu populaire », in Mauger (Gérard), Pelletier (Willy), coord., Les classes populaires et le FN. Explication de votes, Vulaines-sur-Seine, Éditions du Croquant, 2016, pp. 117-132.

47. Beaud, Stéphane, Pialoux (Michel), art. cit., p. 87.

48. Eribon (Didier), La société comme verdict..., op.cit., p. 252.

49. «Dans cette perspective, il faudrait se demander, par exemple, ce que ces représentations doivent à la plus ou moins grande proximité entre sociologues et classes populaires : on peut supposer, en effet, que la distance incline aux représentations "théoriques" et/ou "romanesques" et, en définitive, à la mise en scène de "prolétariats imaginaires". À l'inverse, la proximité n'exclut pas l'ethnocentrisme : ainsi l'extension donnée à l'univers à géométrie variable des classes populaires dépend vraisemblablement de la plus ou moins grande proximité par rapport aux fractions "établies" ou "marginales" des classes populaires et des affinités ou des préjugés associés à cette proximité » (Mauger, $2013: 11$ ).

50. Mauger (Gérard), «De "l'homme de marbre" au "beauf". Les sociologues et "la cause des classes populaires" ", Savoir/Agir, n² 26, 2013, p. 12.

51. https://www.lesinrocks.com/2018/12/04/actualite/edouard-louis-chaque-personne-quiinsultait-un-gilet-jaune-insultait-mon-pere-111149208/.

52. Esslin (Martin), Bertolt Brecht ou les pièges de l'engagement, Paris, UGE, 1971, p. 195. 
53. Jean-Paul Sartre critiquait déjà, au mitan des années 1950, le TNP de Jean Vilar, auquel il reprochait de ne pas avoir de public véritablement populaire.

54. De l'individualisme méthodologique et du post-structuralisme dans le domaine académique.

55. Neveux (Olivier), Théâtres en lutte. Le théâtre militant en France des années 1960 à aujourd'hui, Paris, La Découverte, 2007, p. 146.

56. Ibid., p. 239.

57. «J'ai suffisamment dénoncé l'oubli des classes populaires par les gauches gouvernementales pour ne pas accepter aujourd'hui qu'on nous demande à l'inverse d'oublier, au nom d'un "commun" totalisant et homogénéisant, et donc autoritaire et réactionnaire, et souvent même, je le répète, quasi fascisant, toutes les luttes que l'on appelle - pour les disqualifier - "identitaires", ou "particularistes". Or ces luttes sont toutes aussi légitimes les unes que les autres. Mais elles ne se croisent pas nécessairement. Chacune défend des intérêts spécifiques » (D. Eribon - https:// www.theatredelaville-paris.com/files/e2e6d79e/retour_a_reims_pedago.pdf).

58. Bensaïd (Daniel), Les irréductibles. Théorèmes de la résistance à l'air du temps, Paris, Textuel, 2001, p. 41 .

INDEX

Mots-clés : Didier Eribon, Retour à Reims, Thomas Ostermeier, socio-analyse, Théâtre

\section{AUTEUR}

\section{FABIEN GRANJON}

Sociologue, Fabien Granjon est professeur en Sciences de l'éducation à l'Université Paris 8, membre du laboratoire Experice 